\title{
Top US universities, institutes for life sciences in 2014
}

\section{Brady Huggett}

Using a ranking of US research institutions based on five years (2010-2014) of total gross licensing revenue reported by the Association of University Technology Managers (AUTM), Nature Biotechnology obtained detailed life science commercialization information for 2014, then re-ranked these major universities by number of licenses/options executed (Table 1). Biomedical research institutes are found in Table 2. The number of licenses executed from this group have jumped considerably (566 for 10 universities in 2013; 1,072 for 11 in 2014), life science startups also grew (93 for 10 schools in 2013; 136 for 11 in 2014). Life sciences remain the driving force behind startup creation for this group (Fig. 1), with New York University sitting atop our list of license income earners (Fig. 2).

\begin{tabular}{|c|c|c|c|c|c|}
\hline University & Licenses and/or options executed & Gross licensing revenue received (\$) & Startups & NIH awards ${ }^{a}$ & NIH funding $(\$)^{\mathrm{a}}$ \\
\hline Univ. of California System & 223 & $109,253,453$ & 52 & 4,134 & $1,780,390,316$ \\
\hline Univ. of Washington/Wash. Res. Fdn. & 222 & $51,547,500$ & 13 & 933 & $427,465,936$ \\
\hline Duke Univ. & 124 & $34,117,861$ & 4 & 730 & $350,742,239$ \\
\hline Univ. of Minnesota & 119 & $21,900,000$ & 9 & 550 & $248,295,819$ \\
\hline Univ. of Pennsylvania & 103 & $17,000,000$ & 11 & 1,106 & $484,421,830$ \\
\hline Univ. of Utah & 71 & $73,770,453$ & 12 & 381 & $145,539,936$ \\
\hline Columbia Univ. & 68 & $162,000,000$ & 12 & 874 & $351,353,417$ \\
\hline Univ. of Illinois Chicago Urbana & 49 & $22,630,000$ & 5 & 314 & $114,689,235$ \\
\hline New York Univ. & 42 & $212,317,461$ & 5 & 593 & $301,329,508$ \\
\hline Univ. of Florida & 31 & $25,699,314$ & 6 & 313 & $129,743,178$ \\
\hline California Inst. of Technology & 20 & $3,485,259$ & 7 & 138 & $63,082,330$ \\
\hline
\end{tabular}

*MIT, Stanford, University of Wisconsin-Madison, and the University of Texas system also ranked highly in gross licensing revenue, but could not provide information specific to life sciences. Source: AUTM,

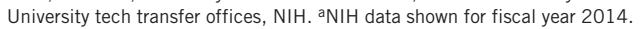

Table 2 US biomedical research institutes ranked by licenses executed, together with revenue, startups and NIH awards and funding in 2014.

Research institute

\begin{tabular}{lllll} 
Licenses and/or options executed & Gross licensing revenue received $(\$)$ & Startups & NIH awards* & NIH funding $(\$)^{*}$ \\
\hline 105 & $68,906,000$ & 6 & 776 & $350,015,302$ \\
\hline 94 & $41,497,523$ & 7 & 401 & $201,234,282$ \\
\hline 67 & $9,375,921$ & 6 & 563 & $321,608,586$ \\
\hline 42 & $164,769,901$ & 1 & 244 & $113,567,614$ \\
\hline 33 & $7,328,686$ & 3 & 304 & $124,995,490$ \\
\hline 32 & $24,114,089$ & 0 & 210 & $122,813,873$ \\
\hline 32 & $5,847,365$ & 4 & 240 & $108,156,442$ \\
\hline 28 & $16,246,038$ & 4 & 195 & $80,948,724$ \\
\hline 24 & $3,712,937$ & 2 & 79 & $40,510,332$ \\
\hline
\end{tabular}

The General Hospital dba Massachusetts General Hospital

Mayo Fdn. for Medical Education and Research 9

Brigham \& Women's Hospital Inc. 6

Memorial Sloan Kettering Cancer Center

Children's Hospital Boston 33

Dana-Farber Cancer Inst.

Beth Israel Deaconess Medical Ctr.

Cleveland Clinic

The Salk Inst. for Biological Studies

St. Jude Children's Research Hospital

Source: AUTM, NIH. *NIH data from fiscal year 2014

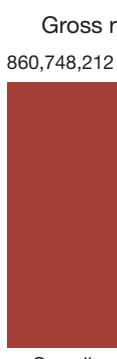

Overall
Licenses executed

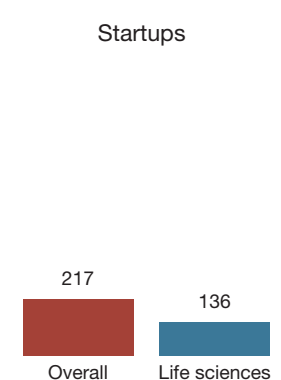

Figure 1 The life sciences accounted for the majority of incoming revenue, outgoing licenses and startups in 2014. Data from universities in Table 1.

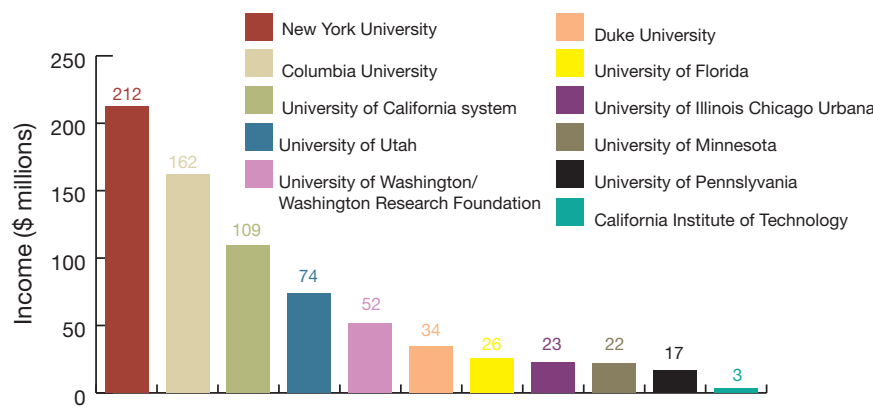

Figure 2 Life science licensing income earners, 2014.

Brady Huggett is business editor at Nature Biotechnology. 